\title{
Bedside echocardiography in internal medicine: which are the key questions and answers for our decision-making?
}

\author{
Chiara Cogliati, ${ }^{1}$ Daniela Torzillo, ${ }^{1}$ Francesco Casella, ${ }^{1}$ Marta Del Medico, ${ }^{1}$ Nicola Montano ${ }^{2}$ \\ ${ }^{1}$ Department of Internal Medicine, L. Sacco Hospital, Milano; ${ }^{2} \mathrm{Ca}$ ' Granda Foundation IRCCS, Maggiore Hospital, University \\ of Milano, Italy
}

\begin{abstract}
The advent of portable equipment in the last years has brought ultrasound (US) technology available at patient bedside, giving the opportunity to non-cardiologists to extend cardiac assessment based on physical examination. Bedside echocardiography is a question-driven examination, where simple and often dichotomous answers are searched. It is performed using phased-array probes and bi-dimensional images are visually evaluated to obtain information regarding cardiac size and function, presence of pericardial effusion, gross valvular diseases. Although this approach cannot in any case substitute a standard 2D color-Doppler echocardiography, bedside echocardiography has been demonstrated to maintain a good diagnostic accuracy when limited to basic questions, even in the hands of short-trained non cardiologist physicians. At present the bedside US approach is widely used in different settings and focused echocardiography takes part together with US explorations of lung, abdomen and deep veins in an integrated perspective that perfectly fits with the holistic approach of the internist. In this context we address two typical scenarios encountered in the internal medicine divisions - the patient presenting with dyspnea or nonpost-traumatic hypotension - showing the main questions we can ask to bedside echocardiography for a rapid identification of the determinants of symptoms and consequently for a therapeutic choice based on more objective evidence.
\end{abstract}

\section{Introduction}

Echocardiography is one of the most requested examinations required by internist for in-hospital patients. This is not surprising taking into account that heart failure (HF) is the first discharge diagnosis ${ }^{1,2}$ for our depart-

Correspondence: Chiara Cogliati, Department of Internal Medicine, L. Sacco Hospital, University of Milano, via G.B. Grassi 74, Milano, Italy.

Tel.: +39.02.39042542 - Fax: +39.02 .50319828 .

E-mail: chiaracogliati@fastwebnet.it

Key words: Cardiac ultrasound; pocketsize ultrasound device; point-of-care ultrasound.

Contributions: $\mathrm{CC}$, conception, drafting and final review of the paper; DT, FC and MDM, collection of references and drafting of the paper; NM, conception and final review of the paper. All authors gave their final approval of the version to be published.

Conflict of interest: the authors declare no potential conflict of interest.

Received for publication: 13 July 2015.

Accepted for publication: 31 July 2015.

This work is licensed under a Creative Commons Attribution NonCommercial 4.0 License (CC BY-NC 4.0).

CCopyright C. Cogliati et al., 2016

Licensee PAGEPress, Italy

Italian Journal of Medicine 2016; 10:83-91

doi:10.4081/itjm.2015.623 ments and that the majority of patients with heart failure is admitted to an internal medicine division. ${ }^{3}$ Moreover, the ever-increasing age of population and the consequent complexity of poli-pathologic patients often require an evaluation of cardiac function not only for diagnostic purposes but also for a correct therapeutic approach of non-cardiac pathologies (for example, cardiac involvement of systemic illness, pre-procedural/surgical evaluation, possible cardiotoxic drugs).

The advent of portable equipment in the last years has brought ultrasound (US) technology available at patient bedside, giving the opportunity to non-cardiologists to extend cardiac assessment based on physical examination. Bedside echocardiography (also reported as focused echocardiography or point of care echocardiography) is a clinical approach performed to answer specific questions arising from anamnesis and physical examination: ${ }^{4}$ significantly enlarged cardiac chambers, moderately or severely reduced left and right ventricular (LV and RV) function, detection of pericardial effusion and signs of tamponade, inferior vena cava size and dynamics can be directly recognized mainly in the perspective of presence/absence of gross abnormalities. Bedside echocardiography of course cannot substitute a standard examination, where high competence is required to grade pathologies and to diagnose even mild abnormalities. Nevertheless, it is out of doubt that bedside echocardiography represents a huge step forward toward a diagnosis as compared to physical examination. ${ }^{5-7}$

The American Society of Echocardiography re- 
cently published international evidence-based recommendations for focused cardiac US. ${ }^{8}$ Most of the evidence of the clinical benefit of the use of bedside echocardiography have been collected in the emergency setting,, 910 and in particular in patients presenting with undifferentiated chest pain, acute dyspnea or shock. ${ }^{11}$ Nevertheless, at present the bedside US approach is widely used in different settings and focused echocardiography takes part together with US explorations of lung, abdomen and deep veins in an integrated perspective that perfectly fits with the holistic approach of the internist. ${ }^{12}$

In this review we summarize the fundamental information we can obtain from cardiac ultrasound performed at bedside and how these information can be compounded by the internist to deal with two major challenges in the daily practice: the dyspnoic patient and the hypotensive patient. Even though this paper is focused on cardiac ultrasound, other applications of bedside ultrasound will be mentioned briefly in some sections, to emphasise the clinical relevance of an integrated ultrasound approach.

\section{Equipment and technique}

Bedside echocardiographic evaluation is usually performed using portable or pocketsize machines carrying a cardiac phased array probe (1.7-3.8 $\mathrm{MHz}$ ). While portable devices allow M-Mode, B-Mode, PW, $\mathrm{CW}$ and color Doppler modalities, pocketsize devices usually support only B-Mode and color Doppler.

All the acoustic windows (parasternal long and short axis, apical 4-chamber, 2-chamber and 3-chamber, subcostal 4-chamber and inferior vena cava views) may be used for cardiac visualization. Nevertheless, when referring to focused echocardiography, it should be taken into account that parasternal view has been demonstrated to be correctly obtained even by short trained physician ${ }^{13}$ while the apical approach requires more expertise because the correct position on the thorax is widely variable and off-axis positioning may lead to uncorrected interpretation of inadequate images. In the critic or unconscious patient the subcostal is often the only accessible approach and it allows simultaneous evaluation of heart and inferior vena cava (IVC).

\section{The bedside cardiac examination}

\section{Detecting alteration of cardiac chambers dimensions}

Detection of significantly enlarged cardiac chamber at bedside is mainly based on visual inspection: loss of normal proportions is recognized and few basic two-dimensional (2D) measurements can be performed as confirmation (Figure 1). ${ }^{14,15}$
Left ventricle dilation is often associated with chronic systolic dysfunction. A rough bi-dimensional diameter $>55 \mathrm{~mm}$ can be assumed as a cut off for dilation if measurements are correctly taken orthogonally to LV walls and just below the mitral plane. Body size of the patient must be obviously taken into account and measurements might be repeated in different scans.

Bedside evaluation of significant LV hypertrophy is based on visual recognition of increased wall thickness; if bi-dimensional measurements are performed as confirmation, a wall thickness of $10 \mathrm{~mm}$ can be considered as upper normal limit.

Right ventricular dimensions are better estimated at end-diastole from a 4-chamber apical view. Being highly dependent on probe position and rotation, to avoid overestimation of right ventricular dimensions the transducer should be positioned over the cardiac apex with the plane through the left ventricle in the center of the cavity. To avoid underestimation, the transducer is rotated until the maximal plane is obtained.

An assessment of right ventricular dilation on bedside examination should include a qualitative evaluation of right ventricular size as compared to LV size. A right ventricular dilation is present when right ventricle appears greater than left ventricle on a 4-chamber apical view $(\mathrm{RV} / \mathrm{LV}$ ratio $>1)$. Moreover a right ventricular basal diameter greater than $42 \mathrm{~mm}$ on a 4chamber apical view can be defined as the cut-off for right ventricular dilation (Figure 1).

Dilation of left atrium can be appreciated visually, comparing it with aortic root in parasternal long axis view. A diameter $>40 \mathrm{~mm}$ or an apical 4 chamber area $>20 \mathrm{~cm}^{2}$ define left atrial dilation. ${ }^{14}$ Right atrium is normally smaller than left atrium; its area measured in apical 4-chambers view is considered normal when $<18 \mathrm{~cm}^{2,15}$ (Figure 1).

Aortic root and ascending aorta are better visualized in parasternal long axis view. Significant dilation/aneurism of ascending aorta $(45 \mathrm{~mm})$ can be visually detected; nevertheless, aortic dissection is not included in international recommendation for focused echocardiography needing its recognition a higher level of competence. ${ }^{4}$

\section{Inferior vena cava diameter and dynamics}

Inferior vena cava is visualized from the subcostal view. From the short axis IVC entrance in the right atrium is appreciated and it is possible to evaluate the changes in diameter related to respiratory phases: in normal conditions reduction in intra-thoracic pressure during inspiration determines an increase in venous inflow accompanied by the decrease in diameter of IVC. Thus, evaluation of IVC diameter and dynamics allows an estimation of right atrial pressure. ${ }^{16}$ In particular, IVC diameter $<21 \mathrm{~mm}$ (measured at a distance of $\approx 2 \mathrm{~cm}$ from right atrium entrance) with collapse 
with sniff $>50 \%$ is associated with right atrial pressure $\leq 5 \mathrm{mmHg}$; IVC diameter $>21 \mathrm{~mm}$ with a collapse with sniff $<50 \%$ identifies elevated atrial pressure (15 $\mathrm{mmHg}$ and higher $)^{15}$ (Figure 1).

\section{Recognition of ventricular systolic dysfunction}

The global left ventricle systolic function is judged as normal or depressed through the inspective evaluation of walls' systolic movement and thickening. ${ }^{11,17,18}$ The acoustic windows recommended for bedside cardiac ultrasound are the parasternal long and short axis and apical 4-chambers views. ${ }^{19}$

Recognition of left ventricle regional wall motion abnormalities requires a higher level of competence and is considered unlikely to be detected by bedside echocardiography.

The assessment of global systolic function of the right ventricle should be performed through the inspective appreciation of the systolic reduction in ventricular area.

\section{Detecting significant left side valve abnormalities}

Two-dimension imaging can identify gross aortic or mitral valve diseases. In particular very calcific aortic or mitral valve with reduced excursion of leaflets allow inferring aortic ${ }^{20}$ or mitral stenosis. A flail mitral flap reveals a significant mitral regurgitation. ${ }^{21}$
In our experience, a visual semi-quantification of regurgitation may be obtained through evaluation of jet dimensions and pattern at color-Doppler (Figure 2). A significant ( $\geq$ moderate) mitral regurgitation may be suspected when wide, large color jets extend deep into the atrium, showing a mosaic pattern. Evaluation of regurgitant jet requires at least two different views. Particular attention has to be paid when considering eccentric regurgitation, where of course the jet area hugging the atrial wall can be under-represented.

Similarly, color jet deepening into the ventricle, showing a mosaic pattern and being wide at the entrance of outflow tract may identify a significant aortic regurgitation (Figure 2).

\section{Pericardial effusion}

Pericardial effusion appears as a black-anechoic space between the heart and the pericardial sheet. It can be totally echo-free or can have echogenic septa or humps inside, as often observed in long lasting or in hematic/infected pericardial effusion.

A small anechoic space in the inferior region during systole is an absolutely non-pathological finding, being a small amount of serous fluid $(15-35 \mathrm{~mL})$ physiologically present in the pericardium. Vice versa, when an echo-free space is visible in diastole the diagnostic suspect of a pericardial effusion can be confirmed.

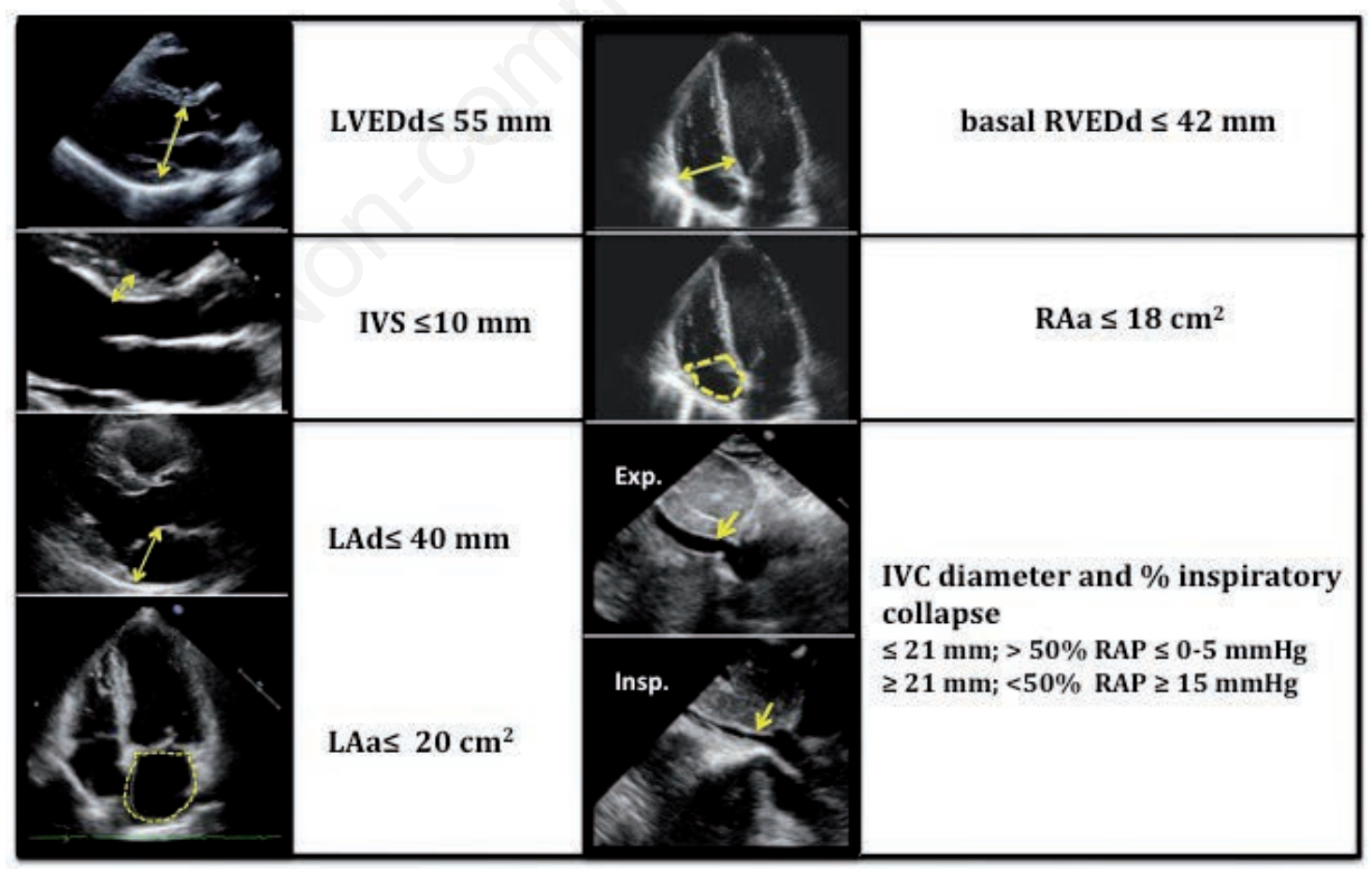

Figure 1. Two-dimension normal values of cardiac chambers and inferior vena cava. LVEDd, left ventricular end diastolic diameter; RVEDd, right ventricular end diastolic diameter; IVS, interventricular septum; RAa, right atrial area; LAd, left atrial diameter; IVC, inferior vena cava; RAP, right atrial pressure; LAa, left atrial area. 
Semi-quantitative estimation of pericardial effusion is obtained measuring the maximum width of the black space, i.e., the maximum distance between the two pericardial sheets: pericardial effusion is defined as small when the separation between parietal and visceral pericardium is less than $10 \mathrm{~mm}$, moderate between 10 and $20 \mathrm{~mm}$, large when $>20 \mathrm{~mm}$. Measurements have to be performed during ventricular and atrial diastole. ${ }^{22}$

Signs of tamponade include diastolic right chambers collapse, an interventricular septum bulge into the left ventricle and inferior vena cava plethora.

Low-pressure chambers are of course the first to be interested by an increased pressure in the pericardial space. Diastolic right atrium collapse has a high positive predictive value for cardiac tamponade when it is present during all the cardiac cycle. Right ventricular diastolic collapse has a good accuracy in predicting cardiac tamponade. ${ }^{22}$

\section{Diagnostic accuracy}

Bedside cardiac ultrasound imaging allows a reliability and accuracy comparable to standard echocardiography to evaluate cardiac dimensions, significant systolic dysfunction and pericardial effusion ${ }^{7,23,24}$ even when performed by non cardiologist $\mathrm{t}^{25,26}$ or medical residents ${ }^{6,27}$ after a short training period and using miniaturized ultrasound devices.

Although there is still no consensus among experts on utilization of color-Doppler for bedside evaluation, some authors have shown at least moderate reproducibility for miniaturized devices in the recognition of significant valvular disease. ${ }^{24}$ Moreover despite the obvious limits of a gross evaluation, different studies showed that bedside echocardiography is definitely superior to physical examination in the detection of valvular regurgitation. ${ }^{28,29}$

Heterogeneous results regarding the modality and duration of training for bedside echocardiography have been obtained through different teaching protocols applied to medical students or residents.

\section{Integrating cardiac ultrasound}

\section{Approaching the dyspnoic patient}

Dyspnea is one of the most common symptoms to deal with in an internal medicine division.

The evaluation of the patient with dyspnea fore-
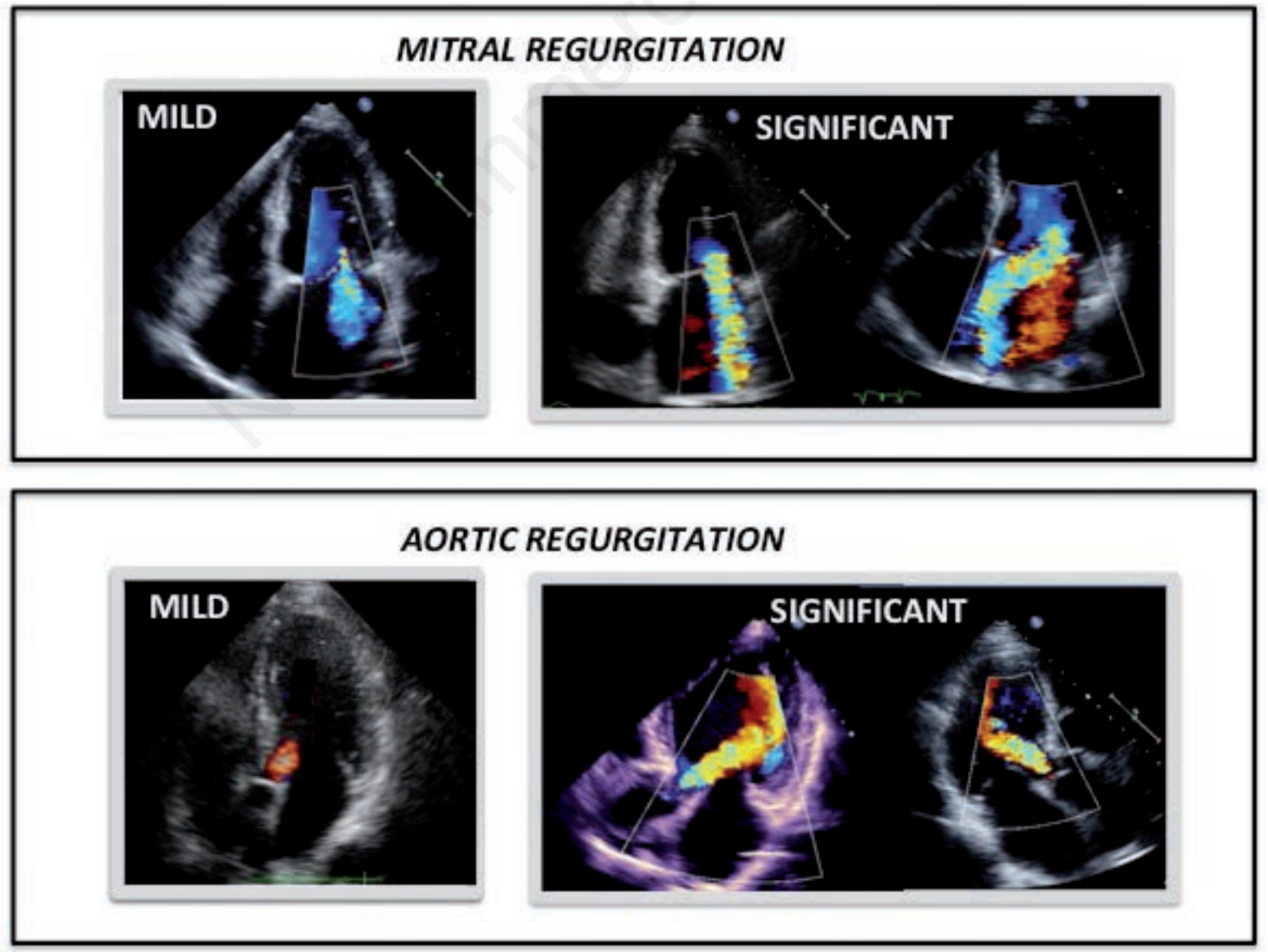

Figure 2. Visual 2D color-Doppler evaluation of mitral and aortic regurgitation in apical view: examples of mild and significant regurgitation. Notice the wide and mosaic-like pattern jets in significant mitral and aortic regurgitation. 
sees a diagnostic algorithm where the lungs, the heart and inferior vena cava are evaluated combining an integrated bedside ultrasound approach with physical examination and radiologic and clinical data. ${ }^{30}$

\section{The lung: wet or dry?}

In the last years lung ultrasound has become a useful diagnostic tool in the hands of the clinicians and a lot of evidence has been collected regarding its accuracy in the differential diagnosis of dyspnea. ${ }^{31}$ The presence of multiple, diffuse and bilateral ultrasound pulmonary artifacts called B-lines (interstitial pattern) and a normal pleural line identify a wet lung, featuring a cardiogenic dyspnea. In the dyspnoic patient in the emergency setting diffuse interstitial pattern has been demonstrated to be highly predictive for cardiogenic dyspnea. ${ }^{32,33}$ In the presence of a wet lung cardiac ultrasound evaluation it will be obviously the next step, mainly looking for a left ventricle dysfunction or for a significant disease of mitral or aortic valve (Figure 3).

The left ventricle: is it dilated? Is there a significant systolic dysfunction? Are there signs of diastolic dysfunction? Is there a severe mitral or aortic valve dysfunction? Is there a pericardial effusion?

Recognition of a dilated left ventricle and/or a significant decreased systolic function may directly identify the cause of dyspnea in patients with signs of lung imbibition.

Nevertheless left ventricular diastolic dysfunction has to be considered in the case of a preserved systolic function. Patients with systolic preserved heart failure represent almost the half of heart failure population, they are older, more frequently women and commonly with comorbidities, a quite common subject admitted to internal medicine division.

Diastolic dysfunction assessment and quantification represent a real challenge even for experienced echocardiographers. Nevertheless, bedside US cardiac evaluation can be useful in call upon it, first of all using an exclusion principle: in the presence of a dyspnoic patient with a wet lung and a preserved systolic function where gross mitral and aortic disease are excluded, diastolic dysfunction remains the most reasonable cause of cardiogenic dyspnea. Moreover, in the same conditions, the detection of a left atrial enlargement can indicate an increased filling pressure while left ventricle hypertrophy, when present, may suggest the diagnosis.

Evaluation of aortic and mitral leaflets for exclusion of gross motion defects and color-Doppler identification of significant regurgitation will allow excluding gross valvular dysfunction.

Pericardial effusion may manifests with dyspnea.

While it is important to explore the heart from all the views in case of suspected pericarditis - when even small quantities of fluid can confirm a diagnostic suspect - in the dyspnoic patient the subcostal view is usually the best approach. Semi-quantification of fluid and evaluation of the compressive effect on cardiac chambers can be easily obtained together with inferior vena cava dynamics (Figure 3).

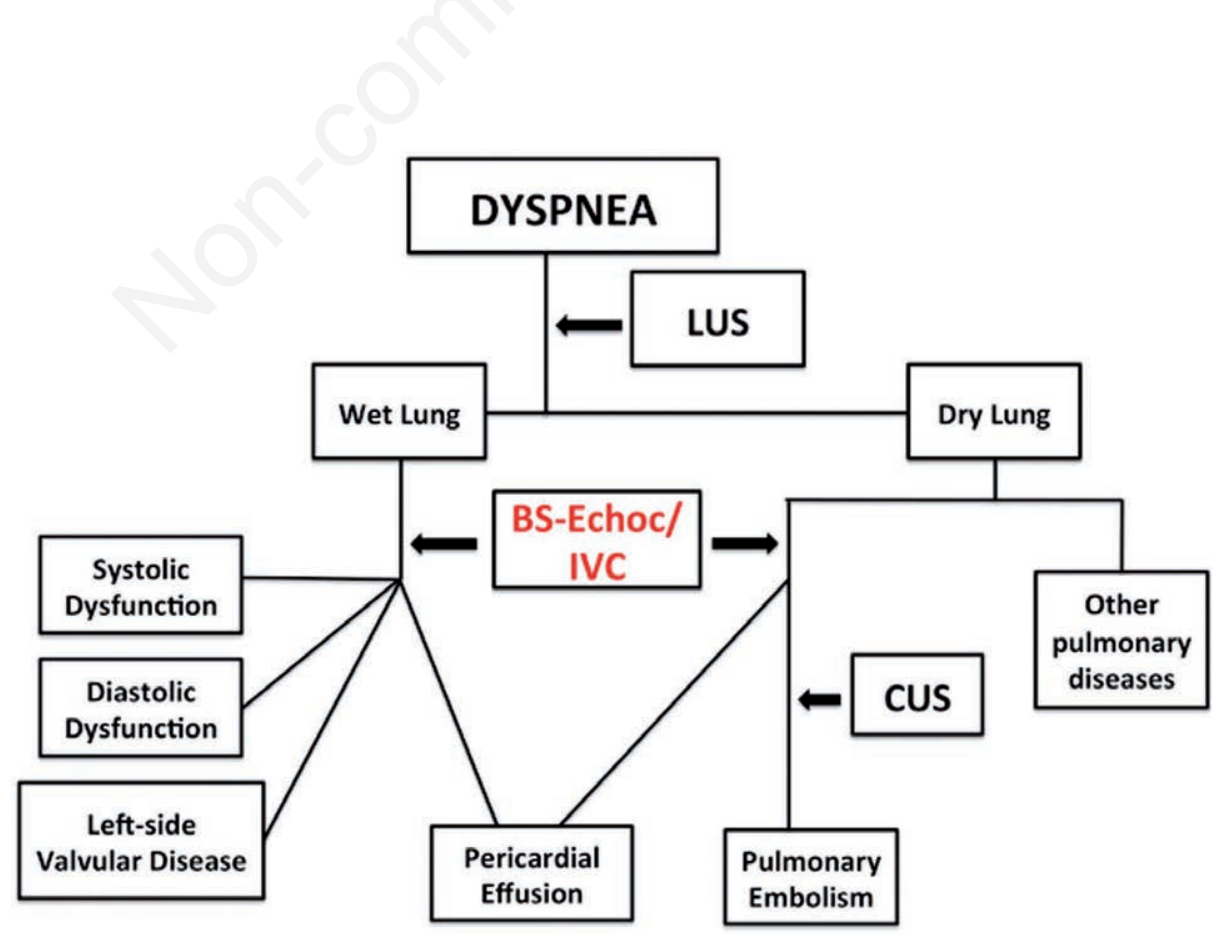

Figure 3. Example of the diagnostic algorithm of dyspnea using bedside integrated ultrasound [lung/heart/inferior vena cava (IVC)]. LUS, lung ultrasound; BS-Echoc, bedside echocardiography; CUS, compressive ultrasound. 
The patient is dyspnoic but the lung is dry: are there signs of acute cor pulmonale?

When a dry lung with preserved pleural sliding is observed, pulmonary embolism should be suspected (Figure 3). Echocardiographic signs of right ventricular overload have a poor diagnostic accuracy in diagnosing acute pulmonary embolism mainly because of a low sensitivity: echocardiographic signs of right ventricular failure are not found in a significant percentage of hemodynamically stable patients. For this reason echocardiography alone is not useful in ruling-out pulmonary embolism in this group of patients. ${ }^{34}$ Moreover, right ventricular dilation and dysfunction are not specific of pericardial effusion and may be found in other cardiac and pulmonary diseases leading to pulmonary hypertension such as left-sided heart failure and chronic obstructive pulmonary disease (COPD) ${ }^{35}$

Venous compressive US may be a useful tool in patients with suspected pulmonary embolism (Figure 3). In this setting the presence of a deep venous thrombosis significantly increases the probability of pulmonary embolism. ${ }^{36}$

Finally, despite the low diagnostic accuracy of single-organ ultrasound examinations, it is has been recently demonstrated that integrated lung/cardiac/ IVC/compressive US allow a good sensitivity and specificity. ${ }^{35}$

\section{Approaching the hypotensive patient}

\section{Is it a cardiogenic shock?}

Shock and hypotension are common clinical conditions in internal medicine and emergency department and are associated with significant mortality rate.

The availability of portable ultrasound device is changing not only the diagnostic approach but also the management of shock: examination can be repeated to assess the evolution and the response to therapy. There are many observational studies showing that bedside cardiac ultrasound improves the diagnostic accuracy and management of shock. A randomized clinical trial observed that immediate goal-directed ultrasound for patients with undifferentiated hypotension in emergency department leads clinicians to significantly narrow their differential diagnosis. ${ }^{10}$ Furthermore bedside ultrasound was shown to increase Emergency Department physicians' certainty in the treatment of hemodynamic impairment of septic shock. ${ }^{37}$

In patients with hypotension a state of volume depletion can be easily identified when a small collapsing IVC, a small hyperkinetic left ventricle and a dry lung are observed (Table 1).

It is important here to underline that IVC diameter and dynamics have to be interpreted taking into account the overall complexity of the hemodynamic state of the patient. In the presence of chronically increased arterial pulmonary pressure (as can be observed in $\mathrm{HF}, \mathrm{COPD}$ or other chronic pulmonary pathologies) IVC can be normal in diameter and collapse even during hypovolemia. Thus, an integrated approach involving cardiac and pulmonary ultrasound is mandatory.

Cardiac causes of hypotension can be diagnosed at the bedside. Beside the recognition of a severe left ventricle systolic dysfunction or a severe valvular disease, as described above, life-threatening conditions such as pericardial tamponade and acute cor pulmonale can be recognized (Table 1). Although, as stated above, aortic dissection is considered out of the competence of bedside cardiac examination, accompanying signs can be recognized and suspicion can be inferred.

\section{Is there evidence of left ventricular dysfunction? Are there signs of valvular dysfunction?}

In patients with undifferentiated hypotension an evaluation of global left ventricular function is carried out to determine whether any part of the patient's

Table 1. Integration of bedside echocardiography with lung and inferior vena cava ultrasound in the non-traumatic hypotensive patient.

\begin{tabular}{|c|c|c|c|c|c|c|}
\hline & Hypovolemic shock & Cardiogenic shock & Cardiac tamponade & Acute cor pulmonale & Pneumotorax & Septic shock \\
\hline Heart & $\begin{array}{l}\text { Small-hyperkinetic } \\
\text { LV/walls kissing }\end{array}$ & $\begin{array}{l}\text { Dilated-hypokinetic } \\
\text { LV/aortic or mitral } \\
\text { disease }\end{array}$ & Pericardial effusion & $\begin{array}{l}\text { Dilated-hypokinetic } \\
\text { RV }\end{array}$ & $\begin{array}{l}\text { Small-hyperkinetic } \\
\text { LV/walls kissing }\end{array}$ & $\begin{array}{l}\text { Small-hyperkinetic } \\
\text { LV/walls kissing } \\
\text { or } \\
\text { Sepsis-relted } \\
\text { systolic dysfunction } \\
\text { Septic source: } \\
\text { endocarditis }\end{array}$ \\
\hline Lung & Dry & Wet & $\begin{array}{l}\text { Dry or wet/ } \\
\text { associated } \\
\text { pleural effusion }\end{array}$ & Dry & Dry/no sliding & $\begin{array}{l}\text { Dry/ARDS } \\
\text { Septic source: } \\
\text { lung consolidation }\end{array}$ \\
\hline IVC & Small-collapsing & $\begin{array}{l}\text { Dilated/ } \\
\text { non-collapsing }\end{array}$ & $\begin{array}{l}\text { Dilated/ } \\
\text { non-collapsing }\end{array}$ & $\begin{array}{l}\text { Dilated/ } \\
\text { non-collapsing }\end{array}$ & $\begin{array}{l}\text { Dilated/ } \\
\text { non-collapsing }\end{array}$ & Small/collapsing \\
\hline
\end{tabular}

$\mathrm{LV}$, left ventricular; RV, right ventricular; ARDS, acute respiratory distress syndrome; IVC, inferior vena cava. 
shocked state may be explained by poor left sided cardiac function (Table 1). The rapid determination of left ventricular function may lead to different management strategies of patients with undifferentiated hypotension. The pattern of end-systolic effacement of the left ventricle walls mandates volume resuscitation, whereas a severe LV dysfunction cautions against major volume resuscitation in favor of inotropic support rather than vasopressor use.

Bedside recognition of a significant mitral or aortic valve dysfunction allows guiding the therapeutic approach, being treatment of hypotension potentially quite different depending on the valvular defect.

\section{Are there signs of pericardial tamponade?}

A pericardial effusion was found to be present in a significant percentage of patients presenting to Emergency Department with unexplained hypotension. ${ }^{38}$ The prevalence was seen to rise to $40 \%$ in a small population of patients with near pulseless electric activity states. ${ }^{39}$ Once a pericardial effusion is identified, determining its contribution to hemodynamic instability is required. In addition to clinical data, the clinician may seek out findings of either right atrial or ventricular diastolic collapse and IVC plethora to support a diagnosis of tamponade (Table 1).

\section{Are there signs of acute cor pulmonale?}

As previously mentioned echocardiographic signs of right ventricular overload have a poor diagnostic accuracy in diagnosing acute pulmonary embolism in hemodinamically stable patients. Vice versa, in the hypotensive patients the absence of echocardiographic signs of right ventricular failure excludes pulmonary embolism as a cause of hemodynamic instability. ${ }^{40}$

\section{Are there signs of possible aortic dissection?}

Regardless of the visualization of the intimal flap, transthoracic echocardiography is useful in detecting aortic root dilation, aortic regurgitation and pericardial effusion, which are usually associated with ascending aorta dissection. ${ }^{41}$ A recent study showed that bedside echocardiography may be a useful triage tool in suspected type A aortic dissection to rapidly identify patient who may benefit from urgent aortic imaging examination and surgical intervention; however it is not reliable as a single test to rule in or rule out type $\mathrm{A}$ aortic dissection. ${ }^{42}$

\section{Is the shock state fluid-responsive?}

One of the priorities in the treatment of shock is fluid resuscitation, which may raise cardiac output and restore critical organ perfusion. However many patients fail to respond to fluids and an indiscriminate fluid therapy increases the risk of edema. A key deci- sion in the management of shock is to identify patients who may benefit from volume resuscitation and when fluid infusion has to be stopped.

Hyperkinetic left ventricle and a small inferior vena cava size can predict fluid responsiveness in spontaneously breathing patients.

Moreover, an integrated bedside ultrasound helps clinicians to assess the evolution of the illness and the response to therapy. First, inferior vena cava size can be frequently reassessed providing feedback on the effects of each intervention and guiding the next steps in management. Furthermore lung ultrasound can detect fluid overload at an early and subclinical stage. The presence of extensive B-lines on lung ultrasound is both sensitive and specific for interstitial syndrome and might contraindicate further volume resuscitation. ${ }^{43}$

\section{Is the cause of the shock state other than cardiac in origin?}

Bedside cardiac ultrasound is useful even when the etiology of shock is other than cardiac in origin (Table 1).

Hypovolemic shock can be strongly suspected when a hyperkinetic left ventricle and a small collapsing IVC are observed.

Sepsis has broad influences on ventricular function ranging from a scenario with high cardiac output and low systemic vascular resistance (sepsis-hyperdynamic state) to a pattern of low cardiac output and myocardial depression ${ }^{44}$ (Table 1). In patients with septic shock bedside echocardiography may be useful to differentiate patients who might benefit from different therapy in addiction to fluid resuscitation (vasopressors for those with hyperdynamic state and inotropes for those with myocardial depression). When the source of bacteremia is not clear, an integrated bedside approach with lung and abdominal ultrasound may help to detect the origin of sepsis. For example the evidence of a pulmonary consolidation on lung ultrasound points to a pulmonary origin of sepsis, while the presence of gallbladder wall thickening and cholelitiasis supports the diagnosis of cholecystitis.

A tension pneumothorax is a life-threatening condition associated with hemodynamic instability and a sonographic pattern of hyperkinetic left ventricle and large non-collapsing caval vein. Lung ultrasound has a good diagnostic accuracy in diagnosing tension pneumothorax..$^{45}$

\section{Conclusive considerations}

Bedside ultrasound represents a very powerful diagnostic tool that of course has to be considered not as an alternative but as an extension of the basic clinical approach of which anamnesis and physical examination represent the cornerstones. 
The technological improvement has brought in particular bedside echocardiography to be extensively applied, dramatically changing the classic semeiotic.

If there are no doubts that many medical schools all over the world are introducing ultrasonography in their curriculum, a systematic effort is needed from academic institutions and scientific societies to standardize training programs for a minimum competence acquisition. ${ }^{46,47}$

\section{References}

1. Kellett J, Deane B. The diagnoses and comorbidity encountered in the hospital practice of acute internal medicine. Eur J Intern Med 2007;18:467-73.

2. Duckitt R, Palsson R, Bosanska L, Dagna L, et al. Common diagnoses in Internal Medicine in Europe 2009: a pan-European, Multi-Centre Survey. Eur J Intern Med 2010;21:449-52.

3. Di Lenarda A, Scherillo M, Maggioni AP, et al. Current presentation and menagement of heart failure in cardiology and internal medicine hospital units: a tale of two worlds - The TEMISTOCLE study. Am Heart J 2003; 146:E12.

4. Spencer KT, Kimura BJ, Korcarz CE, et al. Focused cardiac ultrasound: recommendations from the American Society of Echocardiography. J Am Soc Echocardiogr 2013;26:567-81.

5. Giannotti G, Mondillo S, Galderisi M, et al. Hand-held echocardiography: added value in clinical cardiological assessment. Cardiovasc Ultrasound 2005;3:7.

6. Galderisi M, Santoro A, Versiero M, et al. Improved cardiovascular diagnostic accuracy by pocket size imaging device in non-cardiologic outpatients: the NaUSiCa (Naples Ultrasound Stethoscope in Cardiology) study. Cardiovasc Ultrasound 2010;8:51.

7. Cardim N, Fernandez Golfin C, Ferreira D, et al. Usefulness of a new miniaturized echocardiographic system in outpatient cardiology consultations as an extension of physical examination. J Am Soc Echocardiogr 2011;24: 117-24.

8. Via G, Hussain A, Wells M, et al. International evidencebased recommendations for focused cardiac ultrasound. J Am Soc Echocardiogr 2014;27:1-33.

9. Levitt MA, Jan B. The effect of real time 2-D -echocardiography on medical decision-making in the emergency department. J Emerg Med 2002;22:229-33.

10. Jones AE, Tayal VS, Sullivan DM, Kline JA. Randomized, controlled trial of immediate versus delayed goaldirected ultrasound to identify the cause of nontraumatic hypotension in emergency department patients. Crit Care Med 2004;32:1703-8.

11. Labovitz AJ, Noble VE, Bierig M, et al. Focused cardiac ultrasound in the emergent setting: a consensus statement of the American society of Echocardiography and American College of Emergency Physicians. J Am Soc Echocardiogr 2010;23:1225-30.

12. Arienti V, Camaggi V. Clinical applications of bedside ultrasonography in internal and emergency medicine. Intern Emerg Med 2011;6:195-201.

13. Hellmann DB, Whiting-O'Keefe Q, Shapiro EP, et al. The rate at which residents learn to use hand-held echocardiography at the bedside. Am J Med 2005;118:1010-8.

14. Lang RM, Bierig M, Devereux RB, et al. Recommendations for chamber quantification: A report from the American Society of Echocardiography's Guidelines and Standards Committee and the Chamber Quantification Writing Group, developed in conjunction with the European Association of Echocardiography, a branch of the European Society of Cardiology. J Am Soc Echocardiogr 2005;18:1440-63.

15. Rudski LG, Lai WW, Afilalo J, et al. Guidelines for the echocardiographic assessment of the right heart in adults: a report from the American society of Echocardiography. J Am Soc Echocardiogr 2010;23:685-713.

16. Brennan JM, Blair JE, Goonewardena S, et al. Reappraisal of the use of inferior vena cava for estimating right atrial pressure. J Am Soc Echocardiogr 2007;20:857-61.

17. Kimura BJ, Amundson S, Willis CL, et al. Usefulness of a hand-held ultrasound device for bedside examination of left ventricular function. Am J Cardiol 2002; 90:1038-9.

18. Melamed R, Sprenkle MD, Ulstad VK, et al. Assessment of left ventricular function by intensivists using handheld echocardiography. Chest 2009;135:1416-20.

19. Mark DG, Ku BS, Carr BG, et al. Directed bedside transthoracic echocardiography: preferred cardiac window for left ventricular ejection fraction estimation in critically ill patients. Am J Emerg Med 2007;25:894-900.

20. Abe Y, Ito M, Tanaka C, et al. A novel and simple method using pocket-sized echocardiography to screen for aortic stenosis. J Am Soc Echocardiogr 2013;26:589-96.

21. Shmueli H, Burstein Y, Sagy I, et al. Briefly trained medical students can effectively identify rheumatic mitral valve injury using a hand-carried ultrasound. Echocardiography 2013;30:621-6.

22. Klein AL, Abbara S, Agler DA, et al. American Society of Echocardiography clinical recommendations for multimodality cardiovascular imaging of patients with pericardial disease: endorsed by the Society for Cardiovascular Magnetic Resonance and Society of Cardiovascular Computed Tomography. Am Soc Echocardiogr 2013;26:965-1012.

23. Prinz C, Voigt JU. Diagnostic accuracy of a hand-held ultrasound scanner in routine patients referred for echocardiography. J Am Soc Echocardiogr 2011;24:111-6.

24. Andersen GN, Haugen BO, Graven T, et al. Feasibility and reliability of point-of-care pocket-sized echocardiography. Eur J Echocardiogr 2011;12:665-70.

25. Melamed R, Sprenkle MD, Ulstad VK, et al. Assessment of left ventricular function by intensivists using handheld echocardiography. Chest 2009;135:1416-20.

26. Michalski B, Kasprzak JD, Szymczyk E, Lipiec P. Diagnostic utility and clinical usefulness of the pocket echocardiographic device. Echocardiography 2012;29:1-6.

27. Razi R, Estrada JR, Doll J, Spencer KT. Bedside handcarried ultrasound by internal medicine residents versus traditional clinical assessment for the identification of systolic dysfunction in patients admitted with decompensated heart failure. J Am Soc Echocardiogr 2011;24:1319-24.

28. Kobal SL, Trento L, Baharami S, et al. Comparison of effectiveness of hand-carried ultrasound to bedside cardiovascular physical examination. Am J Cardiol 2005;96: 1002-6. 
29. Stokke TM, Ruddox V, Sarvari SI, Otterstad JE. Brief group training of medical students in focused cardiac ultrasound may improve diagnostic accuracy of physical examination. J Am Soc Echocardiogr 2014;27: 1238-46.

30. Laursen CB, Sloth E, Lambrechtsen J, et al. Focused sonography of the heart, lungs and deep veins identifies missed life-threatening conditions in acute admitted patients with respiratory symptoms. Chest 2013;144: 1868-75.

31. Volpicelli G, Elbarbary M, Blaivas M, et al. International evidence-based recommendations for point-ofcare lung ultrasound. Intens Care Med 2012;38:577-91.

32. Lichtenstein DA, Mezière GA. Relevance of lung ultrasound in the diagnosis of acute respiratory failure the BLUE protocol. Chest 2008;134:117-25.

33. Cibinel GA, Casoli G, Elia F, et al. Diagnostic accuracy and reproducibility of pleural and lung ultrasound in discriminating cardiogenic causes of acute dyspnea in the emergency department. Intern Emerg Med 2012;7:65-70.

34. Roy P, Colombet I, Durieux P, et al. Systematic review and meta-analysis of strategies for the diagnosis of suspected pulmonary embolism. BMJ 2005;331:259.

35. Haddad F, Doyle R, Murphy DJ, Hunt SA. Right ventricular function in cardiovascular disease, Part II: pathophysiology, clinical importance, and management of right ventricular failure. Circulation 2008;117:1717-31.

36. Le Gal G, Righini M, Sanchez O, et al. A positive compression ultrasonography of the lower limb veins is highly predictive of pulmonary embolism on computed tomography in suspected patients. Thromb Haemost 2006;95:963-6.

37. Mansencal N, Vieillard-Baron A, Beauchet A, et al. Triage patients with suspected pulmonary embolism in the emergency department using a portable ultrasound device. Echocardiography 2008;25:451-6.

38. Haydar SA, Moore ET, Higgins GL, et al. Effect of bedside ultrasonography on the certainty of physician clinical decisionmaking for septic patients in the emergency department. Ann Emerg Med 2012;60:346-58.

39. Mandavia DP, Hoffner RJ, Mahaney K, Henderson SO. Bedside echocardiography by emergency physicians. Ann Emerg Med 2001;38:377-82.

40. Tayal VS, Kline JA. Emergency echocardiography to detect pericardial effusion in patients in PEA and nearPEA states. Resuscitation 2003;59:315-8.

41. Kucher N, Luder CM, Dörnhöfer T, et al. Novel management strategy for patients with suspected pulmonary embolism. Eur Heart J 2003;24:366-76.

42. Meredith EL, Masani ND. Echocardiography in the emergency assessment of acute aortic syndromes. Eur J Echocardiogr 2009;10:31-9.

43. Nazerian P, Vanni S, Castelli M, et al. Diagnostic performance of emergency transthoracic focus cardiac ultrasound in suspected acute type A aortic dissection. Intern Emerg Med 2014;9:665-70.

44. Lichtenstein D. FALLS-protocol: lung ultrasound in hemodynamic assessment of shock. Heart Lung Vessel 2013;5:142-7.

45. Antonucci E, Fiaccadori E, Donadello K, et al. Myocardial depression in sepsis: From pathogenesis to clinical manifestations and treatment. J Crit Care 2014;29:500-11.

46. Soldati G, Testa A, Sher S, et al. Occult traumatic pneumothorax: diagnostic accuracy of lung ultrasonography in the emergency department. Chest 2008;133:204-11.

47. Solomon SD, Saldana F. Point-of-care ultrasound in medical education - stop listening and look. N Engl J Med 2014;370:1083-5. 